\title{
ISÓTOPOS ESTABLES DEL CARBONO EN PATAGONIA MERIDIONAL: DATOS DE LA CUENCA DEL LAGO SALITROSO (PROVINCIA DE SANTA CRUZ, REPÚBLICA ARGENTINA)
}

\author{
AUGUSTO TESSONE ${ }^{1}$, A. FRANCISCO ZANGRANDO ${ }^{2}$, GUSTAVO BARRIENTOS ${ }^{2}$ \\ SUSANA VALENCIO ${ }^{3}$, HÉCTOR PANARELLO $^{3}$ Y RAFAEL GOÑI $^{4}$
}

En memoria de Susana Valencio:

"Mientras este trabajo estaba en prensa hemos sufrido la pérdida irreparable de Susi Valencio. Los autores de este trabajo, al igual que el resto de nuestros colegas, la extrañaremos siempre por sus excelentes cualidades como persona y como profesional. Este trabajo está dedicado a ella".

\section{RESUMEN}

En este artículo se presentan y discuten los resultados de estudios de isótopos estables del carbono efectuados sobre restos óseos humanos pertenecientes a 25 individuos procedentes de la localidad Sierra Colorada (cuenca del lago Salitroso), entre los cuales 19 han sido datados directamente usando ${ }^{14} \mathrm{C}$-AMS (ca. 2750 a 300 años Cal. AP). A partir de dicha información, exploramos la composición de la dieta durante el Holoceno tardío y, a nivel intra-poblacional, comparamos los valores de $\delta^{13} \mathrm{C}$ con las categorías de sexo y edad identificadas en la muestra. Asimismo, examinamos las propiedades químicas, elementales e isotópicas de las muestras óseas analizadas con el fin de evaluar el grado de preservación de la señal isotópica primaria y de medir su utilidad como indicador de paleodieta. En lo que respecta a la ecología isotópica, aportamos nuevos valores de $\delta^{13} \mathrm{C}$ de muestras de guanacos y vegetales de la zona. Los resultados obtenidos ratifican la importancia de los ecosistemas terrestres en la dieta de estas poblaciones y la baja o nula incorporación de recursos marinos.

PALABRAS CLAVES: Isótopos estables del carbono, Dieta, Holoceno tardío.

\section{CARBON STABLE ISOTOPES IN PATAGONIA MERIDIONAL: DATA FROM THE SALITROSO LAKE BASIN (SANTA CRUZ PROVINCE, ARGENTINA)}

\section{ABSTRACT}

This work presents and discusses the carbon isotope data obtained from the analysis of human bone remains from 25 individuals of the locale of Sierra Colorada (Salitroso lake basin), 19 of which have been dated directly by

1 INAPL - 3 de Febrero 1378 (CP1426), Buenos Aires, Argentina. E-mail: gutitessone@yahoo.com.ar

2 CONICET-AIA / UBA - Rivadavia 1379 11ํF (CP1033), Buenos Aires, Argentina. E-mail: panchozan@yahoo.com.ar CONICET-INAPL / UNLP/ UNCPBA - 3 de Febrero 1378 (CP1426), Buenos Aires, Argentina.

E-mail: barrient@museo.fcnym.unlp.edu.ar

3 CONICET-INGEIS - Pabellón INGEIS, Ciudad Universitaria (CP1428), Buenos Aires, Argentina.

E-mail: susana@ingeis.uba.ar; hector@ingeis.uba.ar

4 UBA - INAPL - 3 de Febrero 1378 (CP1426), Buenos Aires, Argentina. E-mail: rgoni@filo.uba.ar 
${ }^{14} \mathrm{C}$-AMS to between ca. 2750 and 300 cal. BP. This information enables us to examine diet composition through the Late Holocene and to compare the $\delta^{13} \mathrm{C}$ values within sex and age categories at intra-population level. We have also examined the chemical, elemental, and isotopic properties of bone samples in order to evaluate the degree of preservation of the primary isotopic signal and their suitability as palaeodiet indicator. As a contribution to isotope ecology, we supply new $\delta^{13} \mathrm{C}$ values for local samples of plants and guanacos. The results obtained highlight the importance of terrestrial ecosystems in the diet of these populations and their low or negligible intake of marine resources.

KEY WORDS: Carbon stable isotopes, Diet, Late Holocene.

\section{INTRODUCCIÓN}

El objetivo de este trabajo es presentar la información actualmente disponible de valores de isótopos estables del carbono de muestras humanas, faunísticas y vegetales provenientes de la cuenca del lago Salitroso y otras áreas del noroeste de la provincia de Santa Cruz (Fig. 1). Es de destacar que, en ambientes terrestres con predominio de vegetales $\mathrm{C}_{3}$ como es el caso de Patagonia meridional (Paruelo et al. 1998), los isótopos estables del carbono cons- tituyen una herramienta confiable para evaluar la composición de paleodietas humanas (Tauber 1981; Chisholm et al. 1982; Walker y DeNiro 1986).

En particular, se discutirá esta información en el marco del modelo de poblamiento propuesto para la región bajo estudio (Goñi y Barrientos 2000; Goñi et al. 2000-2002 y Goñi y Barrientos 2004). Este modelo sostiene que las condiciones climáticas y ambientales cambiantes durante el Holoceno tardío habrían afectado la movilidad y el tamaño de las poblaciones cazadoras-recolectoras.

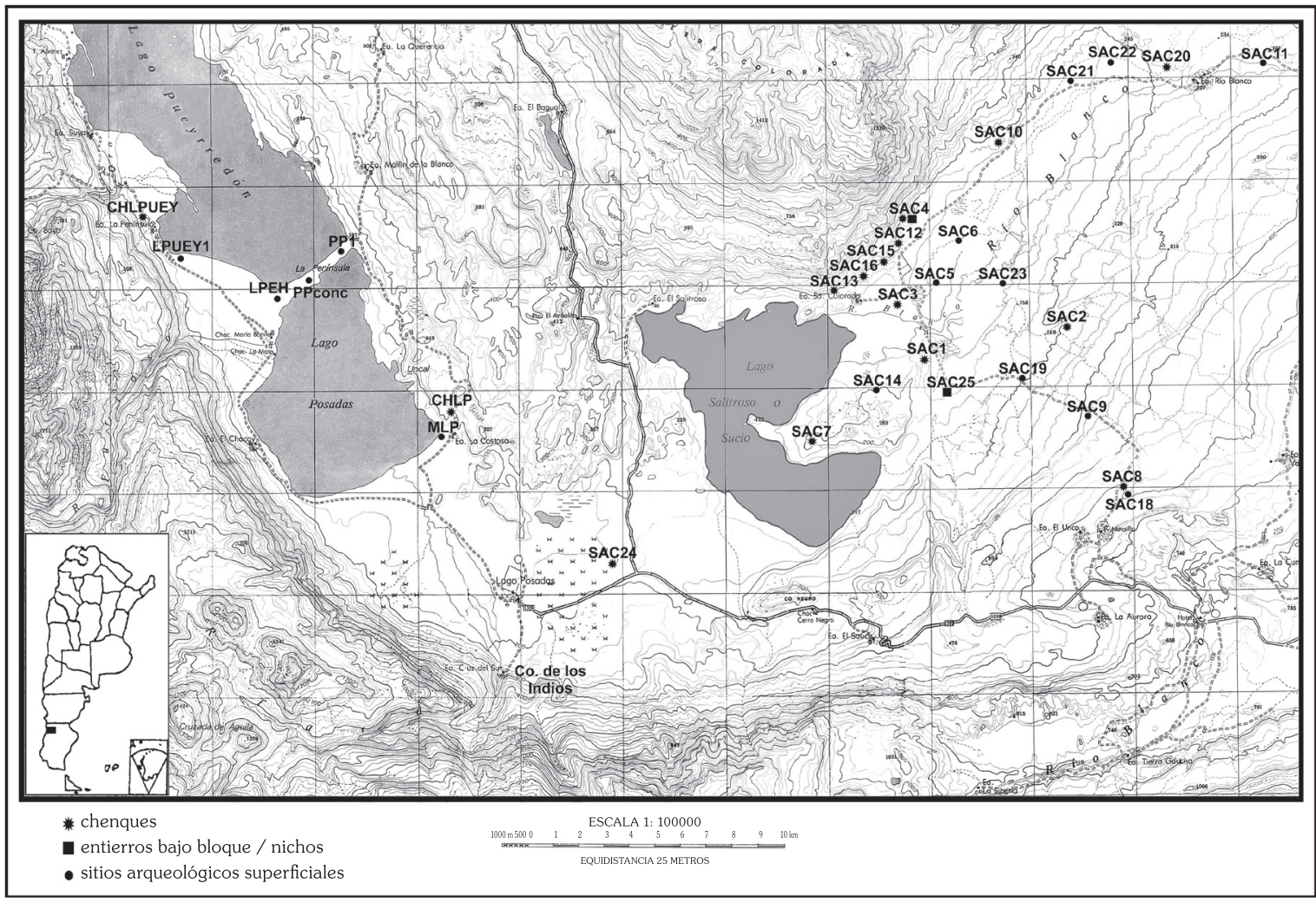

Fig. 1. Mapa de ubicación de la zona de estudio donde se observan los entierros y sitios arqueológicos presentes en la cuenca del lago Salitroso (Goñi y Barrientos 2004). 
La creciente aridización vinculada con la Anomalía Climática Medieval (ca. 1150 a 600 años Cal. AP; Stine 1994) habría producido un patrón de ocupaciones prolongadas y con una redundancia en el uso de espacios con disponibilidad de recursos críticos (v.g. agua, leña, etc.) combinado con un aprovisionamiento logístico de recursos animales y vegetales. Partiendo de las hipótesis propuestas por este modelo, se evalúan las posibles tendencias temporales en el registro isotópico y su correlato con las variaciones en la subsistencia y en la movilidad de estas poblaciones cazadoras-recolectoras.

Si bien la creciente utilización de isótopos estables en Patagonia meridional ha generado un considerable avance en la comprensión de distintos aspectos de la conducta humana prehistórica (Fernández y Panarello 1996; Borrero et al. 2001; Barberena 2002; entre otros), es necesario destacar que la mayor parte de las muestras humanas analizadas carecen de información cronológica directa, razón por la cual es escaso el conocimiento sobre la distribución temporal de este tipo de evidencia. En este trabajo buscamos contribuir a la discusión de este aspecto inspeccionando la variabilidad a largo plazo en la dieta de las poblaciones cazadorasrecolectoras del área.

\section{EL REGISTRO BIOARQUEOLÓGICO EN EL LAGO SALITROSO}

La cuenca del lago Salitroso presenta una alta densidad de entierros humanos en tres modalidades distintas: chenques, entierros bajo bloque y nichos (Fig. 1). Hasta el momento se han excavado un total de 32 entierros, los cuales están ubicados, a excepción de un caso, en cerros bajos, bardas y otras geoformas elevadas. Los chenques constituyen la forma de entierro más abundante y con mayor distribución en toda la cuenca, mientras que la cantidad y distribución de nichos y entierros bajo bloque es mucho menor (Goñi et al. 2000-2002). Existen claras diferencias estructurales entre estos tipos de entierro. Los chenques son acumulaciones de rocas depositadas intencionalmente, de contorno oval o circular, de entre 3 y 5 metros de diámetro y comúnmente constituyen entierros múltiples. Los nichos y los entierros bajo bloque son pequeños reparos naturales aprovechados para la depositación de restos humanos y presentan en la mayoría de los casos contextos individuales. También se registran diferencias cronológicas entre las formas de entierro. Los chenques se distribuyen temporalmente entre ca. 1200 y 300 años Cal. AP, mientras que la cronología de los nichos y entierros bajo bloque está comprendida entre ca. 2750 y 2250 años Cal. AP. Hasta el presente se ha recuperado un mínimo de 79 individuos, entre los cuales están representadas todas las categorías de sexo y edad (Bernal et al. 2004).

Los valores presentados en este trabajo se obtuvieron sobre restos óseos humanos procedentes de todas las modalidades de entierro: 20 recuperados en chenques, 2 en nichos y 3 en entierros bajo bloque. La muestra analizada comprende un total de 25 individuos, doce femeninos, diez masculinos $y$ tres de sexo indeterminado. Las edades de los mismos oscilan entre 1,5 y 55-59 años, las cuales pueden ser agrupadas en tres categorías etáreas: ocho infantiles (0-12 años), cuatro adolescentes (1324 años) y trece adultos (mayor a 25 años) (Bernal et al. 2004).

\section{ASPECTOS METODOLÓGICOS Y CONFIABILIDAD DE LAS MUESTRAS ANALIZADAS}

La determinación de $\delta^{13} \mathrm{C}$ en vegetales y en el colágeno de restos óseos humanos y de guanacos se llevó a cabo en tres laboratorios: Laboratorio de Isótopos Ambientales, Instituto de Geocronología y Geología Isotópica, Argentina (AIE); Center for Applied Isotope Studies, University of Georgia (UGA) y Arizona Radiocarbon AMS Facility, University of Arizona (AA) (Tablas 1 y 2). En el caso de estas dos últimas instituciones, los valores presentados constituyen los parámetros que se utilizaron para la determinación de la edad radiocarbónica de las muestras. Dado que entre las tres instituciones no se cuenta con intercalibraciones directas, en los restos óseos humanos fue necesario verificar la consistencia de los datos comparando los valores de aquellas muestras que fueron analizadas en por lo menos dos laboratorios. Se observó que, en la gran mayoría de los casos, las variaciones entre los datos están incluidas dentro de la incertidumbre analítica informada por los laboratorios. Cabe consignar que pese a ser, en promedio, superiores los valores de Arizona respecto de los del INGEIS, y de no estar 
ambos conjuntos correlacionados $(r=0,12)$, no existen diferencias estadísticamente significativas entre sus medias (test de $\mathrm{t} ; \mathrm{AA}=-19,3 \% 0 \pm 0,4 \% 0 ; \mathrm{AIE}=$ $-18,9 \% 0 \pm 0,6 \% ; \mathrm{t}=-1,8 ; \mathrm{gl}=24 ; \mathrm{p}=0,083)$. En el INGEIS la determinación de los isótopos estables del carbono se efectuó sobre el colágeno extraído de costillas de cada uno de los individuos $(\mathrm{n}=19)$ utilizando los métodos convencionales: 1) separación de la fracción colagénica de la fracción mineral del hueso y de otras fases orgánicas incorporadas postdepositacionalmente, mediante la técnica descripta por Longin (1971) y con posterioridad modificada por Schoeninger y DeNiro (1984); 2) obtención de $\mathrm{CO}_{2}$ por combustión del colágeno $(10 \mathrm{mg})$ con $\mathrm{V}_{2} \mathrm{O}_{5}(200$ mg) durante ocho horas a $550{ }^{\circ} \mathrm{C}$; 3) purificación criogénica del $\mathrm{CO}_{2}$ y 4) medición de las relaciones isotópicas $\left({ }^{13} \mathrm{C} /{ }^{12} \mathrm{C}\right)$ en un espectrómetro de masas Finnigan MAT de triple colector.

En la expresión de los resultados se utilizó la notación convencional $\delta \%$ respecto de la referencia internacional V-PDB (Gonfiantini et al. 1995).
Para evaluar el estado de preservación de los huesos, se utilizaron parámetros químicos, elementales e isotópicos (Van Klinken 1999).

Entre los indicadores químicos más confiables se encuentra la cantidad de colágeno, expresada como porcentaje en peso, recuperada de un hueso. Un hueso moderno fresco contiene aproximadamente $22 \%$ en peso de colágeno. De acuerdo con Van Klinken (1999), el rango de variación en el contenido de colágeno de un hueso bien conservado es de 0,5\% a 20,0\% en peso para zonas climáticas templadas. En el caso del lago Salitroso, situado en una zona templada fría, los valores obtenidos, con excepción de SAC 1-2-5, se encuentran comprendidos dentro de dicho rango.

La relación carbono/nitrógeno $(\mathrm{C} / \mathrm{N})$ constituye un indicador elemental de diagénesis utilizado recurrentemente en estudios isotópicos (Harrison y Katzenberg 2003; Richards et al. 2003, entre otros). En las muestras analizadas, esta relación se determinó sobre el hueso total (i.e. fracción orgá-

Tabla 1. Datos de edad, sexo, composición isotópica del carbono, cronología y relación $\mathrm{C} / \mathrm{N}$ de cada uno de los individuos estudiados en la cuenca del lago Salitroso.

\begin{tabular}{|l|c|c|c|c|c|c|}
\hline \multicolumn{1}{|c|}{ Sitio } & Edad & Sexo & No Laboratorio & $\delta^{13} \mathrm{C}(\mathrm{V}-\mathrm{PDB})$ & ${ }^{14} \mathrm{C}$ & $\mathrm{C} / \mathrm{N}$ \\
\hline SAC 4 Nicho & A & I & AA38568 & $-19,2$ & $2.607 \pm 41$ & - \\
\hline SAC 1-8-1 & A & F & AA38563 & $-19,8$ & $2.532 \pm 41$ & - \\
\hline SAC 4 Nicho 1 & A & F & AIE11149 & $-19,1$ & $2.520 \pm 40$ & 4,2 \\
\hline SAC 1-8-2 & A & I & AA38562 & $-19,8$ & $2.494 \pm 43$ & - \\
\hline SAC 4-2-3 & $40-44$ & M & AA38566 & $-19,2$ & $2.274 \pm 41$ & - \\
\hline SAC 1-7-1 & $50+$ & M & AIE11150 & $-19,1$ & $1.147 \pm 37$ & 3,7 \\
\hline SAC 1-7-2 & $25-29$ & M & AIE11151 & $-18,2$ & $1.142 \pm 42$ & 3,9 \\
\hline SAC 1-6-1 & $6 \pm 2$ & I & AIE10308 & $-18,8$ & $756 \pm 32$ & 3,8 \\
\hline SAC 4-1-1 & $55-59$ & F & AIE10311 & $-19,4$ & $720 \pm 39$ & 3,5 \\
\hline SAC 10-1-4 & $11 \pm 2,5$ & M & AIE11148 & $-19,6$ & $687 \pm 43$ & 4,1 \\
\hline SAC 1-6-2 & $20-24$ & F & AIE10309 & $-18,5$ & $690 \pm 40$ & 3,6 \\
\hline SAC 10-1-1 & $15-19$ & M & AIE11147 & $-19,8$ & $662 \pm 43$ & 4,0 \\
\hline SAC 1-1-B & $40-44$ & F & AIE11143 & $-19,7$ & $622 \pm 57$ & - \\
\hline SAC 1-6-3 & $20-24$ & F & AIE10310 & $-18,1$ & $539 \pm 46$ & - \\
\hline SAC 1-4-1 & $8,5 \pm 1$ & M & AA38564 & $-20,0$ & $424 \pm 39$ & - \\
\hline SAC 1-2-1 & $3 \pm 1$ & F & AIE 10304 & $-19,1$ & $418 \pm 40$ & 3,6 \\
\hline SAC 1-2-2 & $40-44$ & F & AIE 10305 & $-18,8$ & $389 \pm 40$ & 3,6 \\
\hline SAC 20-3-2 & $45-49$ & M & UGA10623 & $-18,5$ & $380 \pm 40$ & - \\
\hline SAC 1-1-3 & $35-39$ & F & AIE 10303 & $-18,0$ & $352 \pm 40$ & - \\
\hline SAC 1-1-1 & $15 \pm 3$ & M & AIE 10302 & $-18,8$ & & - \\
\hline SAC 1-1-4 & $7 \pm 2$ & M & AIE11144 & $-18,4$ & & - \\
\hline SAC 1-1-6 & A & F & AIE11146 & $-19,4$ & & - \\
\hline SAC 1-1-A & $7,5 \pm 2$ & M & AIE11142 & $-20,1$ & - & 3,6 \\
\hline SAC 1-2-4 & $1,5 \pm 0,5$ & F & AIE 10306 & $-17,9$ & - & - \\
\hline SAC 1-2-5 & $2 \pm 0,6$ & F & AIE 10307 & $-23,0$ & & 4,1 \\
\hline & & & & - & 4,0 \\
\hline
\end{tabular}

$\mathrm{A}=$ Adulto; $\mathrm{M}=$ Masculino; $\mathrm{F}=$ Femenino; $\mathrm{I}=$ Indeterminados. 
nica más fracción inorgánica) y los valores están comprendidos entre 3,5 y 4,2 (Tabla 1). Si bien no se cuenta con referencias en la literatura que especifiquen el rango de valores considerados normales para huesos bien preservados cuando las mediciones se hacen sobre hueso total, en el caso de mediciones efectuadas sobre el colágeno, tales valores se sitúan entre 2,9 y 3,6 (DeNiro 1985; Ambrose y DeNiro 1989). Se debe tener en cuenta que al realizar el análisis sobre el hueso total, el contenido de carbono medido involucra no sólo al carbono de origen orgánico sino también al inorgánico, por lo que la relación $\mathrm{C} / \mathrm{N}$ se vería incrementada respecto de la determinación sobre el colágeno. Por lo expuesto, estimamos que las muestras de SAC presentan un adecuado estado de preservación.

En ambientes terrestres templados, las muestras con valores de $\delta^{13} \mathrm{C}$ del colágeno más empobrecidos que $-22,0 \%$ deberían examinarse cuidadosamente desde el punto de vista de su conservación (Van Klinken 1999). La única muestra que escapa a los límites estipulados por este parámetro es SAC 1-2-5, con un valor de $-23,0 \%$ (Tabla 1 ).

De acuerdo con los parámetros considerados, podemos afirmar que las muestras óseas estudiadas muestran un buen estado de preservación, con excepción de SAC 1-2-5, y que por lo tanto la señal isotópica de sus colágenos se considera primaria.

\section{ECOLOGÍA ISOTÓPICA EN PATAGONIA MERIDIONAL}

Hasta el momento, no se cuenta con información isotópica referida a la fauna de la cuenca del lago Salitroso. Sin embargo, sí hay información proveniente de áreas cercanas como la cuenca del lago Cardiel y la meseta del lago Strobel, donde los valores de $\delta^{13} \mathrm{C}$ de guanacos presentan una media de $-19,5 \% 0 \pm 0,6 \%$ (Tabla 2). Este resultado es más positivo respecto de los valores medios disponibles para otros sectores de Patagonia meridional. En efecto, los cálculos efectuados por Barberena (2002: 75) señalan un valor medio de $-21,3 \%$ (rango $=-24,9 \%$ o a $-19,0 \%$ ) para el sector cordillerano comprendido entre Lago Argentino y Baguales y espacios adyacentes a Cabo Vírgenes. Esta discrepancia sugiere que los parámetros ecológicos para establecer la incidencia de recursos terrestres en dietas humanas no son homogéneos en Patagonia meridional. Existen diferentes causas que producen variación en los valores de $\delta^{13} \mathrm{C}$ en una misma especie. En el caso de los guanacos, se ha señalado un gradiente latitudinal en sentido N-S con una disminución de los valores de $\delta^{13} \mathrm{C}$ en una escala geográfica macrorregional, posiblemente debida a un decrecimiento en la disponibilidad ambiental de vegetales $\mathrm{C}_{4}$ o CAM que pudieran ser incorporados en la dieta de estos animales (Goñi et

Tabla 2. Cronología y composición isotópica del carbono de recursos animales y vegetales del centro y norte de Santa Cruz.

\begin{tabular}{|l|c|c|c|c|}
\hline \multicolumn{1}{|c|}{ Sitio } & Recursos & № Laboratorio & ${ }^{14} \mathrm{C}$ & $\delta^{13} \mathrm{C}(\mathrm{V}-\mathrm{PDB})$ \\
\hline La Siberia 2 & guanaco & UGA10014 & $1710 \pm 40$ & $-18,6$ \\
\hline La Siberia 2 & guanaco & UGA10013 & $1100 \pm 40$ & $-19,0$ \\
\hline Alero del León & guanaco & UGA10009 & $2190 \pm 50$ & $-19,4$ \\
\hline Parapeto 4 & guanaco & UGA08705 & $2010 \pm 50$ & $-19,4$ \\
\hline Alero Gerasin II & guanaco & UGA10017 & $1580 \pm 40$ & $-19,4$ \\
\hline Cerro Pampa 2 & guanaco & UGA10019 & $310 \pm 40$ & $-19,7$ \\
\hline Solís & guanaco & UGA10018 & $1140 \pm 40$ & $-19,8$ \\
\hline Cerro Pampa 2 & guanaco & UGA10020 & $170 \pm 40$ & $-20,6$ \\
\hline & & & & \\
\hline Manuk 1 & carbón & UGA10011 & $6790 \pm 40$ & $-21,7$ \\
\hline Médanos Lago Posadas & carbón & UGA08702 & $770 \pm 30$ & $-22,2$ \\
\hline Alero Gerasin I & carbón & UGA10016 & $3760 \pm 40$ & $-22,5$ \\
\hline Médanos Margen Sur & carbón & UGA10015 & $1860 \pm 40$ & $-22,6$ \\
\hline SAC 11-2-2 & carbón & UGA08706 & $750 \pm 60$ & $-22,6$ \\
\hline Alero del León & carbón & UGA10008 & $3560 \pm 40$ & $-23,6$ \\
\hline Manuk 1 & carbón & UGA10010 & $940 \pm 40$ & $-23,8$ \\
\hline Alero del León & carbón & UGA10007 & $290 \pm 40$ & $-24,2$ \\
\hline Médanos Lago Posadas & carbón & UGA08703 & $560 \pm 90$ & $-24,9$ \\
\hline
\end{tabular}


al. 2000; Barrientos 2001; Barberena 2002). Sin embargo, este factor difícilmente puede dar cuenta de la totalidad de la diferencia observada entre el centro y el norte de Santa Cruz y regiones situadas al sur del río Santa Cruz. En este sentido, también es importante considerar la posibilidad de que la composición de la dieta de los guanacos varíe en relación con diversas condiciones biogeográficas y ecológicas, ya que se ha registrado una diferencia significativa en la dieta de estos camélidos entre áreas de ecotono bosque-estepa y estepas, al menos en el caso de la Isla Grande de Tierra del Fuego (Bonino y Pelliza Sbriller 1991). En suma, esto podría señalar un contexto ecológico heterogéneo para la interpretación de paleodietas humanas en ambientes terrestres y permitiría generar asimismo expectativas diferentes en relación con este aspecto en Patagonia meridional.

Los valores más enriquecidos observados en guanacos provenientes del sector de mesetas y cuencas cercanas al lago Cardiel pueden deberse a que, en bajos salitrosos y en formaciones medanosas con situaciones semipermanentes de sequía, se den condiciones propicias para el crecimiento de gramíneas o pastos salados con vías fotosintéticas $C_{4}$ (Fernández y Panarello 1991, 1996). Entre las especies presentes en la provincia de Santa Cruz con dichas características, se encuentra Distichlis spica, la cual coloniza mallines secos y suelos salinos (Mazzoni y Vázquez 2003). No obstante, consideramos que si bien estos vegetales conforman una posible fuente a partir de la cual los guanacos pueden adquirir cierto enriquecimiento en ${ }^{13} \mathrm{C}$ con

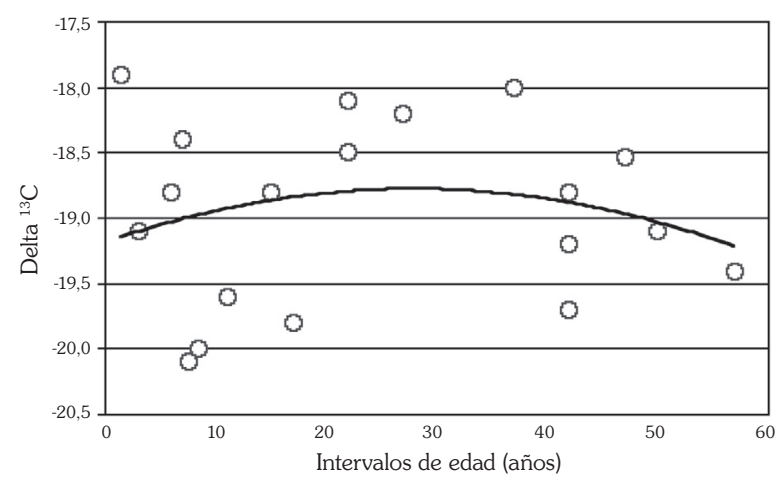

Fig. 2. Distribución de los isótopos estables del carbono en función de los intervalos de edad de individuos recuperados en chenques. respecto a lo observado en otras áreas de Patagonia, la escasa disponibilidad y distribución de estos recursos sugiere una baja incidencia en la dieta de dichos herbívoros. Por otra parte, las muestras correspondientes a plantas arbustivas o leñosas recuperadas en sitios arqueológicos de la región bajo la forma de carbones, presentan valores de $\delta^{13} \mathrm{C}$ comprendidos entre $-21.7 \%$ y $-24.9 \%$ (media $=-23.1 \% 0 \pm 1,0 \%$ ) (Tabla 2). Estos valores sustentan un predominio en el ecosistema de vegetales $C_{3}$.

\section{RESULTADOS Y DISCUSIÓN}

Los resultados de $\delta^{13} \mathrm{C}$ obtenidos en restos óseos humanos procedentes de la cuenca del lago Salitroso, varían entre -17,9\% y -20,1\%o (media = $-19,1 \% 0 \pm 0,7 \% 0$ ) (Tabla 1). La incertidumbre analítica es de $\pm 0,3 \% 0( \pm 2 \sigma)$. Estos valores indican que la dieta de los 25 individuos analizados estuvo basada, fundamentalmente, en el consumo de recursos terrestres vinculados a la vía fotosintética $C_{3}$.

Sólo a partir de un incremento en la disponibilidad de dicha información será posible avanzar hacia reconstrucciones más precisas de dietas humanas en el pasado. Al explorar estos datos en función del sexo de los individuos, no se observan variaciones significativas con respecto a esta variable.

En relación con la edad, el intervalo comprendido entre 0 y 19 años registra la mayor dispersión en los datos isotópicos dentro de la muestra analizada ${ }^{1}$ (Fig. 2). En los individuos con edades entre 20 y 40 años, los registros isotópicos concentran, en término medio, los valores más enriquecidos. En los individuos mayores de cuarenta años, la distribución de valores resulta comparable con la del primer grupo etáreo (Fig. 2). Una explicación probable para las tendencias observadas es la presencia de sesgos de muestreo. No obstante, una hipótesis alternativa es la existencia de diferencias en la dieta entre grupos de edad. Hasta no contar con un tamaño de muestra mayor y con la información brindada por otros parámetros isotópicos (v.g. $\delta^{15} \mathrm{~N}$ ), no es posible evaluar estas interpretaciones con mayor precisión.

Otro punto de discusión consiste en evaluar cómo varió la composición isotópica de la muestra a largo plazo. Los individuos provenientes de nichos

1 En esta evaluación sólo se consideraron individuos recuperados en chenques. 


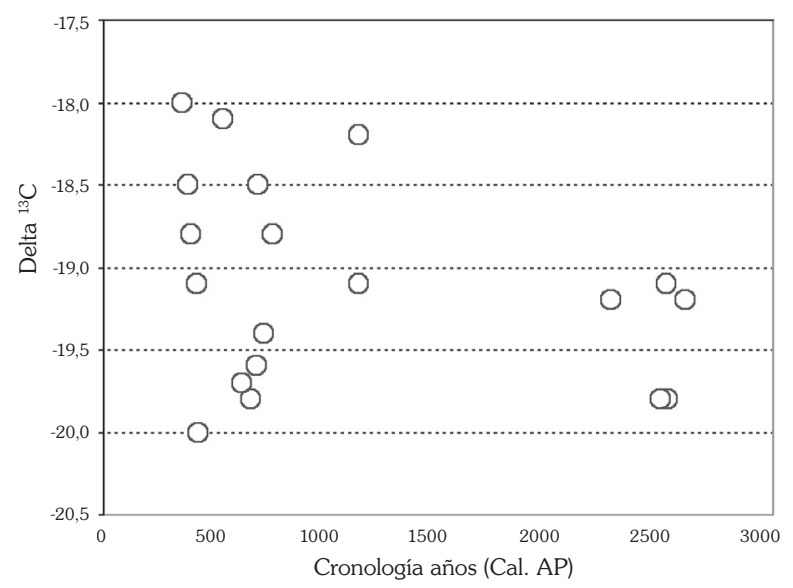

Fig. 3. Variación de $\delta^{13} \mathrm{C}$ de individuos fechados de la cuenca del lago Salitroso en el Holoceno tardío.

$y$ entierros bajo bloque presentan valores de $\delta^{13} \mathrm{C}$ entre $-19,8 \%$ o y $-19,1 \%$ (Tabla 1 ), mientras que los procedentes de chenques exhiben valores entre $-20,1 \%$ y $-17,9 \%$ (Tabla 1). Estas distribuciones indican que no existen diferencias temporales en la dieta, ya que el rango de valores de los entierros más antiguos está subsumido dentro del correspondiente a los entierros más recientes (Fig. 3). A pesar de que otras líneas de evidencia señalan la probable existencia de diferencias temporales en los patrones de movilidad y asentamiento (Goñi et al. 2000-2002), tales variaciones no implicarían cambios substanciales en la dieta, al menos en su componente proteico. Si consideramos a la totalidad de la muestra como un conjunto único, puede decirse que los individuos que conforman el límite superior de la distribución de valores de $\delta^{13} \mathrm{C}$ (Fig. 3) habrían tenido acceso a alimentos de origen marino (Ambrose et al. 1997; Richards y Hedges 1999), si se descarta la posibilidad del consumo directo de vegetales $\mathrm{C}_{4}$ o CAM, suposición justificada en función del conocimiento actual de la ecología regional (Paruelo et al. 1998). No obstante, si nos basamos en la información isotópica correspondiente a guanacos en una escala microrregional, los valores registrados en este sector de Patagonia (Tabla 2) podrían causar el enriquecimiento observado en estos valores humanos. Por otro lado, si bien se espera un papel dominante del guanaco en las paleodietas humanas de la región, es posible que la parte proteica de las mismas también haya sido provista por otros recursos animales poco conocidos en términos isotópicos.
En este sentido, hay que destacar el consumo de choique (Pterocnemia pennata), para el cual se cuenta con una determinación de $\delta^{13} \mathrm{C}$ de $-19,3 \% 0$ (Barberena 2002:75) y de piche (Chaetophractus villosus), especie que aún no es analizada a través de isótopos estables.

En resumidas palabras, la presencia de recursos marinos en la dieta se deberá definir en función de un conocimiento mayor de la ecología isotópica de los potenciales recursos animales y vegetales presentes en Patagonia meridional y de mediciones de otros parámetros isotópicos para esta discriminación (v.g. $\delta^{15} \mathrm{~N}$ ).

\section{CONSIDERACIONES FINALES}

Los isótopos estables del carbono señalan que la dieta de los grupos cazadores-recolectores que habitaron el noroeste de Santa Cruz durante el Holoceno tardío, estuvo basada fundamentalmente en el consumo de recursos terrestres con vías fotosintéticas $\mathrm{C}_{3}$. Por otra parte, la evaluación conjunta de la información cronológica y de $\delta^{13} \mathrm{C}$ indica que no existieron variaciones temporales significativas en la dieta de estas poblaciones. No obstante, se puede proponer una incorporación de recursos marinos en bajas proporciones, ya sea por aprovisionamiento directo o por intercambio, para el final del Holoceno tardío. Esto implicaría que hacia momentos más recientes de este período, los rangos de movilidad podrían haber incluido la costa atlántica y/o pacífica, aunque no en forma recurrente. A nivel intra-poblacional, si bien no se registra variación en función del sexo de los individuos, se observan diferencias en la composición proteica de la dieta con relación a los grupos de edad. Por último, queremos destacar que es imprescindible generar un mayor conocimiento sobre la paleoecología isotópica de Patagonia meridional.

\section{BIBLIOGRAFÍA}

AMBROSE S. H. y M. J. DENIRO. 1989. Climate and habitat reconstruction using stable carbon and nitrogen isotope ratios of collagen in prehistoric herbivore teeth from Kenya. Quaternary Research 31: 407-422.

AMBROSE S.H., B.M. BUTLER, D.B. HANSON, R.L. HUNTER-ANDERSON y H.W. KRUEGER. 1997. Stable isotopic analysis of human diet in the Marianas Archipelago, Western Pacific. American Journal of Physical Anthropology 104: 343-361. 
BARBERENA R. 2002. Los límites del mar: Isótopos estables en Patagonia meridional. Sociedad Argentina de Antropología: Colección Tesis de Licenciatura, 134 pp. Buenos Aires.

BARRIENTOS G. 2001. Estudios de isótopos estables del carbono y nitrógeno en Pampa, Patagonia continental y Tierra del Fuego: una evaluación. Revista Argentina de Antropología Biológica 3 (2): 35.

BERNAL V., S. GARCÍA GURAIEB y P. GONZALES. 2004. Perfiles de mortalidad de las muestras de restos óseos humanos procedentes del área del lago Salitroso (provincia de Santa Cruz). En Contra Viento y Marea. Arqueología de Patagonia: 361-373. Compiladores: Civalero, T., P. Fernández y G. Guraieb. Instituto Nacional de Antropología y Pensamiento Latinoamericano/ Sociedad Argentina de Antropología, Buenos Aires.

BONINO N. y A. PELLIZA SBRILLER. 1991. Composición botánica de la dieta del guanaco (Lama guanicoe) en dos ambientes contrastantes de Tierra del Fuego, Argentina. Ecología Austral 1(2): 97-102.

BORRERO L.A., R.A. GUICHÓN, R.H. TYKOT, J. KELLY, A.I. PRIETO y P. CÁRDENAS. 2001. Dieta a partir de isótopos estables en restos óseos humanos de $\mathrm{Pa}$ tagonia austral. Estado actual y perspectivas. Anales del Instituto de la Patagonia, Serie Ciencias Humanas 29: 119-127.

CHISHOLM B., D. NELSON y H. SCHWARCZ. 1982. Stable carbon isotope ratios as a measure of marine versus terrestrial protein in ancient diets. Science 216: 11311132.

DENIRO M.J. 1985. Postmortem preservation and alteration of in vivo bone collagen isotope ratios in relation to paleodietary reconstruction. Nature 317: 806-809.

FERNÁNDEZ J. y H. PANARELLO. 1991. Paleodietas y patrones de movilidad de cazadores recolectores: su estimación en base a los isótopos estables del carbono. Anales de Arqueología y Etnología 43-45: 599-611.

FERNÁNDEZ J. y H. PANARELLO. 1996. El Rodeo. Análisis de las relaciones isotópicas ${ }^{13} \mathrm{C} /{ }^{12} \mathrm{C}$ en el colágeno. En Contribución a la arqueología del Río Pinturas, provincia de Santa Cruz: 300-310. Eds. Gradin C. y A.M. Aguerre. Ediciones Búsqueda del Ayllu, Concepción del Uruguay.

GONFIANTINI R., W. STICHLER y K. ROZANSKI. 1995. Standards and intercomparison materials distributed by the Internacional Atomic Energy Agency for stable isotope measurements. En Reference and intercomparison materials for stable isotopes of light elements: 13-29. International Atomic Energy Agency-TECDOC825, Vienna.

GOÑI R. y G. BARRIENTOS. 2000. Estudio de chenques en el Lago Salitroso, Provincia de Santa Cruz. En Desde el País de los Gigantes. Perspectivas arqueológicas en Patagonia, Tomo 1: 161-175. Universidad Nacional de la Patagonia Austral, Río Gallegos.

GOÑI R. y G. BARRIENTOS. 2004. Poblamiento tardío y movilidad en la cuenca del Lago Salitroso. En Contra Viento y Marea. Arqueología de Patagonia. Com- piladores: Civalero, T., P. Fernández y G. Guraieb. Instituto Nacional de Antropología y Pensamiento Latinoamericano/ Sociedad Argentina de Antropología, Buenos Aires.

GOÑI R., G. BARRIENTOS, H. PANARELLO, S. VALENCIO y L. LUNA. 2000. Cazadores de Patagonia Austral: aspectos poblacionales y análisis isotópicos. Resúmenes del VI Congreso Latinoamericano de Antropología Biológica. Pirlápolis, Uruguay.

GOÑI R., G. BARRIENTOS y G. CASSIODORO. 2000-2002. Las condiciones previas a la extinción de las poblaciones humanas del sur de Patagonia: una discusión a partir del análisis de la estructura del registro arqueológico de la cuenca del lago Salitroso. Cuadernos del Instituto Nacional de Antropología y Pensamiento Latinoamericano 19: 249-266.

HARRISON R. y A. KATZENBERG. 2003. Paleodiet studies using stable carbon isotopes from bone apatite and collagen: examples from Southern Ontario and San Nicolas Island, California. Journal of Anthropological Archaeology 22 (3): 227-244.

LONGIN R. 1971. New method of collagen extraction for radiocarbon dating. Nature 230: 241-242.

MAZZONI E. y M. VÁZQUEZ. 2003. Ecosistemas de mallines y paisajes de la Patagonia Austral (Provincia de Santa Cruz). Instituto Nacional de Tecnología Agropecuaria, $63 \mathrm{pp}$.

PARUELO J.M., E.G. JOBBÁGY, O.E. SALA, W.K. LAUENROTH y I.C. BURKE. 1998. Functional and structural convergence of temperate grassland and shrubland ecosystems. Ecological Applications 8 (1): 194-206.

RICHARDS M.P. y R.E.M. HEDGES. 1999. Stable isotope evidence for similarities in the types of marine foods used by late mesolithic humans at sites along the Atlantic Cost of Europe. Journal of Archaeological Science 26: 717-722.

RICHARDS M.P., J.A. PEARSON, T.I. MOLLESON, N. RUSSELL y L. MARTIN. 2003. Stable Isotope Evidence of Diet at Neolithic Chatalhöyük, Turkey. Journal of Archaeological Science 30 (1): 67-76.

SCHOENINGER M. y M. DENIRO. 1984. Nitrogen and carbon isotopic composition of bone collagen from marine and terrestrial animals. Geochimica et Cosmochimica Acta 48: 625-639.

STINE S. 1994. Extreme and persistent drought in California and Patagonia during medieval time. Nature 369: 546-549.

TAUBER H. 1981. ${ }^{13} \mathrm{C}$ evidence for dietary habits of prehistoric man in Denmark. Nature 292: 332-333.

VAN KLINKEN G.J. 1999. Bone collagen quality indicators for paleodietary and radiocarbon measurements. Journal of Archaeological Science 26: 687-695.

WALKER P. y M. DENIRO. 1986. Stable nitrogen and carbon isotope ratios in bone collagen as indices of prehistoric dietary dependence on marine and terrestrial resources in Southern California. American Journal of Physical Anthropology 71: 51-61. 